\title{
Eficiência Bionutricional de Animais Nelore e seus Mestiços com Simental e Aberdeen Angus, em Duas Dietas
}

\section{Kepler Euclides Filho1, Valéria Pacheco Batista Euclides², Geraldo Ramos de Figueiredo3, Rodrigo Amorim Barbosa ${ }^{4}$}

\begin{abstract}
RESUMO - Este experimento foi realizado por dois anos com o objetivo de avaliar o desempenho de animais de diferentes potenciais de crescimento,Nelore (N), 1/2 Angus - 1/2 Nelore (AN), 1/2 Simental - 1/2 Nelore (SN). Este estudo faz parte de um projeto amplo, que tem a finalidade de avaliar a eficiência de produção de carne em sistemas de produção compostos por animais de diferentes tamanhos adultos (Projeto Cruzamento Embrapa 1). A partir de uma análise bivariada envolvendo as variáveis ganho de peso e consumo de matéria seca, determinou-se a primeira função discriminante canônica, que foi utilizada para se estimarem índices de desempenho aqui denominados de eficiência bionutricional. Foram avaliados 72 animais em duas dietas, uma constituída de feno de capim-tanzânia mais $600 \mathrm{~g}$ de grão de soja moído fornecido à vontade (dieta A) e outra, constituída de feno adicionado de um concentrado (dieta B). A eficiência bionutricional foi influenciada significativamente pela interação grupo genético (GG) e dieta (D). As comparações entre as médias foram conduzidas pelos contrastes: $\mathrm{C}_{1}$ ) Nelore versus média dos mestiços e, $\mathrm{C}_{2}$ ) 1/2 Angus - 1/2 Nelore versus $1 / 2$ Simental - 1/2 Nelore, na dieta B e; $\mathrm{C}_{3}$ ) Nelore versus mestiços e $\mathrm{C}_{4}$ ) $1 / 2$ Angus - $1 / 2$ Nelore versus $1 / 2$ Simental - 1/2 Nelore na dieta A. Independente da dieta, os animais Nelore apresentaram pior desempenho do que a média verificada para os animais "meio-sangue". As eficiências bionutricionais médias foram 429,74 e 490,46 para Nelore e mestiços, respectivamente, na dieta B e, na mesma seqüência, 299,70 e 376,10, na dieta A. Os desempenhos dos F1s foram dependentes da dieta. Na dieta A, os animais "meio-sangue" Angus-Nelore e Simental-Nelore apresentaram a mesma eficiência bionutricional $(376,10)$, enquanto na dieta B, os 1/2 Angus - 1/2 Nelore foram superiores $(529,84$ versus 451,09).
\end{abstract}

Palavras-chave: alimentação, bovino de corte, cruzamentos, desempenho, eficiência bionutricional

\section{Bio-Nutritional Efficiency of Nellore and Nellore Crosses with Angus and Simmental under Two Diets}

\begin{abstract}
This experiment was carried out for two years with the objective of evaluating the performance of genetic groups with different genetic potentials for growth, such as Nellore (N), $1 / 2$ Angus - 1/2 Nellore (AN) and $1 / 2$ Simmental - $1 / 2$ Nellore (SN). This study is part of an ample project, which has as principal goal, to evaluate the efficiency of production of systems composed by animals with different mature sizes (Projeto cruzamento Embrapa 1). Using a bivariate analysis involving the variables average daily gain and dry matter intake, it was determined the first discriminant canonical function which was used for estimating performance indexes that were denominated bio-nutritional efficiency. The data were obtained from 72 animals under two diets, one composed by Tanzânia hay plus $600 \mathrm{~g}$ of ground soybean (diet A), provided ad libitum, and other where this hay was combined with a concentrate ration (diet B). The bio-nutritional efficiency was significantly affected by the interaction between genetic group (GG) and diet (D). Thus, the least square means were compared by four different contrasts. These contrasts were, on diet $\mathrm{B}: \mathrm{C}_{1}$ ) Nellore versus average of crossbreds and, $\mathrm{C}_{2}$ ) 1/2 Angus - 1/2 Nellore versus $1 / 2$ Simmental - 1/2 Nellore. And on diet $\mathrm{A}: \mathrm{C}_{3}$ ) Nellore versus crossbreds and, $\mathrm{C}_{4}$ ) $1 / 2$ Angus - 1/2 Nellore versus $1 / 2$ Simmental - $1 / 2$ Nellore. The analyses of these contrasts revealed that, independently on diet, Nellore animals had a worst performance than the average observed for the F1s. The average of bio-nutritional efficiencies were equal to 429.74 and 490.46 for Nellore and crossbred animals, respectively, on diet B and, in the same sequence, 299.70 and 376.10 on diet A. Relatively to the F1 animals, their performances were dependent on diet. On diet A, the $1 / 2$ Angus - 1/2 Nellore and $1 / 2$ Simmental - $1 / 2$ Nellore presented the same bio-nutritional efficiency (376.10) while on diet B, the $1 / 2$ Angus - $1 / 2$ Nellore were superiors (529.84 versus 451.09).
\end{abstract}

Key Words: beef cattle, bio-nutritional efficiency, crossbreeding, feeding, performance

\footnotetext{
${ }^{1}$ Engo․- Agro., Ph.D., CREA № 12.153/D - Visto 1.466/MS, Embrapa Gado de Corte, Caixa Postal 154, CEP 79002-970, Campo Grande, MS E.mail: kepler@cnpgc.embrapa.br

2 Engo-.-Agro., Ph.D., CREA № 12.797/D, Embrapa Gado de Corte.

3 Engo-Agro., M.Sc., CREA № 11.753/D - Visto 1.527/MS, Embrapa Gado de Corte.

4 Bolsista da Fundação de Apoio à Pesquisa Agropecuária e Ambiental - Fundapam, Caixa Postal 154, CEP $79002-970$ Campo Grande, MS.
} 


\section{Introdução}

Com o cenário futuro indicando avanço nos sistemas de produção em direção à intensificação, a pecuária de corte tem de ser enfocada como uma atividade empresarial e, como tal, seus objetivos serão, quase sempre, de natureza econômica (WRIGHT, 1971). Apesar de essa visão exigir que se dê atenção a todos segmentos da cadeia produtiva e a suas inter-relações, ela sem dúvida sinaliza para a importância de identificar e incorporar, aos sistemas de produção, estratégias que resultem em aumento de eficiência.

Nesse contexto, a utilização de mestiços (Bos taurus x Bos indicus) tem se constituído em uma alternativa que se encontra em grande expansão no Brasil. No entanto, considerando-se o segmento sistema de produção, a alimentação talvez seja o fator isolado mais importante, uma vez que a sua inadequação em qualidade, quantidade ou custo pode inviabilizar a produção de gado de corte (CEZAR e EUCLIDES FILHO, 1996). Assim, faz-se necessário avaliar alternativas que possibilitem a melhor relação entre custos com a alimentação e o desempenho animal. Nesse sentido, a conversão alimentar e sua relação com a dieta constituem-se em elementos de fundamental importância para rentabilidade do setor.

OLIVEIRA et al. (1991) relataram que a existência de diferenças quanto ao consumo alimentar e à habilidade de digerir alimentos entre Bos taurus, Bos indicus e seus mestiços tem sido motivos de controvérsias. Alguns estudos revelam que a concentração energética da ração exerce influência diferente nos animais das duas espécies. LEDGER et al. (1970) encontraram maior consumo por unidade de peso vivo para taurinos, quando comparado com zebuínos, e comportamento intermediário para os animais mestiços. Dados semelhantes foram encontrados por GONÇALVES et al. (1991), trabalhando com animais Nelore, Holandês, seus cruzamentos com diferentes "graus-de-sangue" e búfalos. Por outro lado, VALADARES FILHO et al. (1987) relataram que zebuínos têm maior capacidade de digerir alimentos fibrosos, devido a sua melhor capacidade de utilização do nitrogênio. Resultados de outros trabalhos, como os de EUCLIDES FILHO et al. (1997), fizeram com que os autores concluíssem não haver diferenças entre grupos genéticos para conversão alimentar.

Além de todos esses fatores que podem influenciar a conversão alimentar ou mesmo a eficiência alimentar, podem ser ainda mencionados os efeitos de idade do animal e sexo. Todavia, é possível existir ainda outro elemento importante nessas avaliações e comparações, que é o fato de tanto a conversão alimentar quanto a eficiência alimentar resultarem, segundo GUIDONI (1994), em índices viesados para estimação do desempenho nutricional. Ainda segundo esse autor, a combinação do consumo de alimento com o ganho de peso, denominado neste trabalho de eficiência bionutricional, resultaria em um valor mais eficiente para avaliar desempenho nutricional. Assim, o objetivo deste trabalho foi avaliar e comparar a eficiência bionutricional de três grupos genéticos, Nelore, $1 / 2$ Simental - $1 / 2$ Nelore e $1 / 2$ Angus - $1 / 2$ Nelore, em duas dietas, considerando-se as variáveis ganho de peso e consumo de alimento como variáveis correlacionadas.

\section{Material e Métodos}

O trabalho foi realizado na Embrapa Gado de Corte, durante dois anos, 1995 e 1996, e faz parte de um projeto amplo, que tem como objetivo geral avaliar a eficiência de sistemas de produção com base em animais de diferentes potenciais de crescimento e tamanho adulto (Projeto cruzamento Embrapa 1). Todos os animais eram machos inteiros com idade inicial de aproximadamente 11 meses e foram desmamados em abril, pemanecendo, desde o nascimento até o início do experimento, em pastagens de Brachiaria decumbens. Em agosto, início do experimento, eles foram vermifugados e distribuídos em baias individuais dotadas de cocho para suplementação mineral, bebedouro e comedouro para fornecimento de água e distribuição de ração, respectivamente. Em cada um dos anos, foram utilizados 36 animais de três grupos genéticos: 12 Nelore (N), 12 1/2 Simental - 1/2 Nelore (SN) e 12 1/2 Angus - 1/2 Nelore (AN), com pesos médios iniciais iguais a 180, 220 e $221 \mathrm{~kg}, 172$, 209 e $224 \mathrm{~kg}$, respectivamente, para o primeiro e segundo anos experimentais. Seis animais de cada grupo genético foram alocados aleatoriamente a um dos dois tratamentos estudados. Os animais "meiosangue" são filhos de touros das duas raças européias acasalados com vacas Nelore. Para atender à premissa básica do projeto, que é avaliar animais de diferentes potenciais de crescimento e tamanho adulto, os touros foram escolhidos com base em seus pesos adultos. Assim, para a raça Angus, esses apresentavam pesos inferiores a $1000 \mathrm{~kg}$ e, para a raça Simental, esses pesos estavam entre $1000 \mathrm{~kg}$ e $1300 \mathrm{~kg}$.

Como o trabalho tinha o intuito de avaliar o desempenho dos animais em condições que se apro- 
ximassem daquelas observadas em condições de pastagens, procurou-se fornecer dietas que possibilitassem ganhos de peso semelhantes aos observados durante os períodos seco de águas em pastagens bem manejadas. Assim, os animais foram submetidos a duas dietas: A) feno de capim-tanzânia mais $600 \mathrm{~g}$ de grão de soja moído e B) feno de capim-tanzânia mais concentrado (65\% de grão de milho triturado, $11 \%$ de farelo de soja, 19,56\% de farelo de algodão, 2,2\% de calcário calcítico, $2,2 \%$ de uréia e $0,04 \%$ de rumensin). A proporção de volumoso para concentrado, na dieta B, foi de 70:30.

É importante ressaltar que a soja moída foi incluída na ração $\mathrm{A}$, com o intuito de elevar o nível protéico dessa dieta, uma vez que a quantidade de PB do feno de tanzânia (4\%) estava inferior ao mínimo necessário para adequada fermentação ruminal, que, segundo MINSON (1990), para pastagens tropicais, é de aproximadamente, 6\%. Nessas condições, a deficiência protéica provoca decréscimos nas taxas de degradação e passagem e, consequentemente, no consumo voluntário. A melhoria da qualidade nutricional, neste caso particular, visou garantir consumo adequado da ração, uma vez que essa era condição imprescindível para as avaliações propostas. Dessa forma, as dietas A e B apresentavam 8 e $11 \%$ de $\mathrm{PB}$, respectivamente.

Antes de se iniciar o experimento, houve um período de adaptação, de 14 dias, dos animais às dietas.

O período experimental foi variável, uma vez que os animais eram abatidos à medida que atingiam uma cobertura de gordura de $5 \mathrm{~mm}$ de espessura, que foi estimada visualmente. Os pesos foram variáveis de acordo com o grupo genético sendo, aproximadamente, entre 440 e $450 \mathrm{~kg}$ para o Nelore, 460 e $470 \mathrm{~kg}$ para AN e 490 e $500 \mathrm{~kg}$ para SN. As rações foram fornecidas em duas porções, a primeira no período da manhã e a segunda no final da tarde. Para a determinação da conversão alimentar, foram anotadas diariamente as quantidades de ração fornecidas, bem como o resto. Os animais foram pesados a cada 28 dias, após jejum prévio de 16 horas. A cada três dias, com base no consumo, ajustava-se a quantidade de ração fornecida procurando possibilitar consumo ad libitum. Para isso, as quantidades fornecidas, diariamente, foram calculadas de modo a garantir que as sobras fossem de, aproximadamente, $10 \%$ do fornecido.

Para análise dos dados, as variáveis ganho de peso e consumo de alimento foram consideradas de forma conjunta, em uma análise bivariada, utilizando-se o procedimento "MANOVA" existente no SAS (1989). Esse procedimento, segundo GUIDONI (1994), se constitui em um método mais sensível para discriminação do efeito de tratamentos sobre o desempenho nutricional do animal do que a conversão alimentar ou a eficiência alimentar. Para complementação da análise, foi utilizada a primeira função discriminante canônica que, segundo HARRIS (1975), citado por GUIDONI (1994), resulta no maior valor para o teste F, considerando-se qualquer combinação linear envolvendo as variáveis analisadas. As comparações entre os valores obtidos pela primeira função discriminante canônica foram realizadas por meio de contrastes.

\section{Resultados e Discussão}

A análise bivariada envolvendo as variáveis ganho de peso (GP) e consumo de matéria seca (CMS) revelou a existência de efeito significativo para a interação entre grupo genético e dieta $(\mathrm{P}<0,05)$ (Tabela 1). Dessa forma, para obtenção da primeira função discriminante canônica, utilizou-se a matriz referente a esta interação, obtida na análise multivariada.

Essa primeira função discriminante aplicada aos dados de ganho de peso e de consumo resulta em

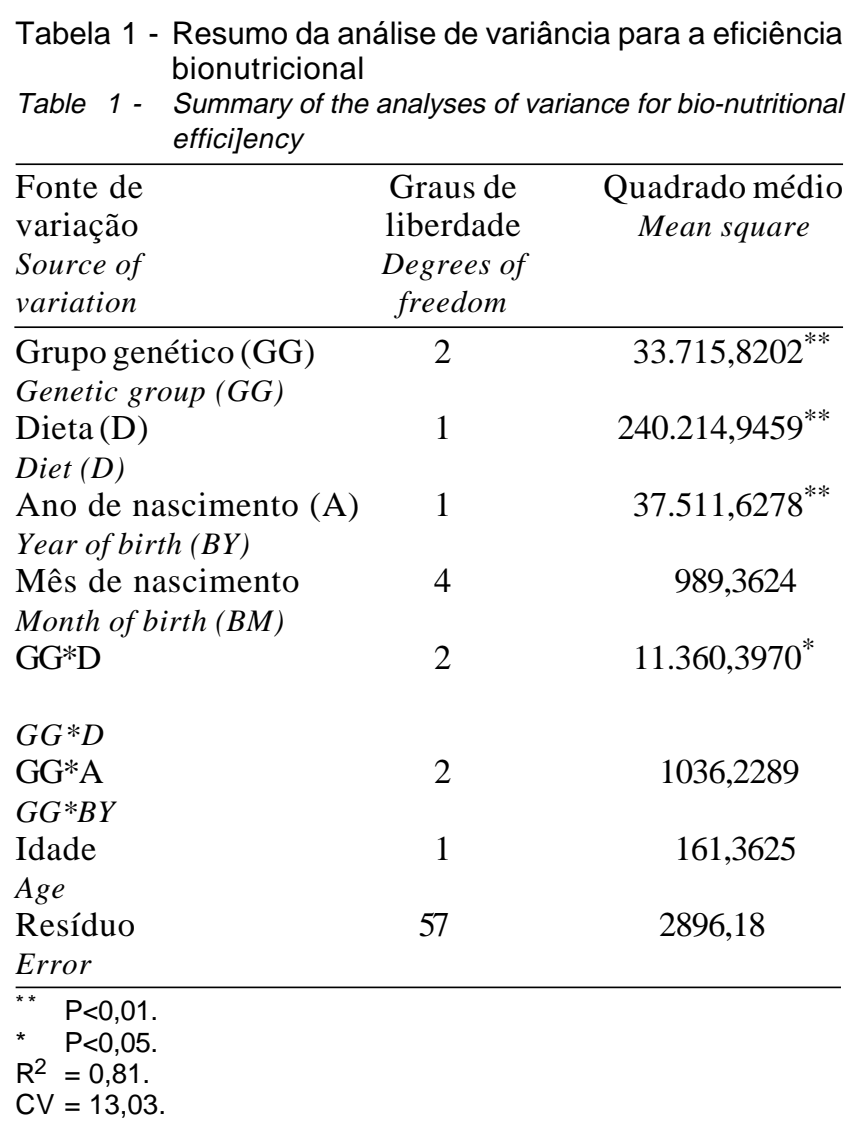


valores que foram denominados, por GUIDONI (1994), de Índice Nutricional Multivariado Biológico. Neste trabalho, como essa função combina ganho de peso e consumo, procurou-se simplificar, denominando-a de Eficiência Bionutricional (EBN). Para sua obtenção, determinou-se inicialmente os autovalores da matriz $\left[\mathrm{E}^{-1} \mathrm{H}\right]$, em que [E] é a matriz do resíduo e $[\mathrm{H}]$, a matriz correspondente à interação GGxD. Utilizando-se o maior autovalor encontrado, foi obtido um autovetor a partir do seguinte sistemas de equações:

$$
\left[\mathrm{E}^{-1}-\lambda \mathrm{I}\right]\left[\begin{array}{l}
\mathrm{a} \\
\mathrm{b}
\end{array}\right]=\left[\begin{array}{l}
0 \\
0
\end{array}\right]
$$

em que 1 é o maior autovetor e "a" e "b" são os coeficientes que serão utilizados na equação da eficiência bionutricional.

Com esse procedimento, determinou-se a seguinte equação para Eficiência Bionutricional:

$$
\mathrm{EBN}=0,9644(\mathrm{GP})-0,2644(\mathrm{CMS})
$$

As médias de quadrados mínimos obtidas para eficiência bionutricional, de acordo com grupo genético e dieta, encontram-se na Tabela 2. Os desempenhos dos diferentes grupos genéticos foram comparados dentro de cada dieta, utilizando-se os seguintes contrastes: $\mathrm{C}_{1}$ ) Nelore versus média dos mestiços e $\mathrm{C}_{2}$ ) $1 / 2$ Angus $-1 / 2$ Nelore versus $1 / 2$ Simental, na dieta $\mathrm{B}$ e $\mathrm{C}_{3}$ ) Nelore versus mestiços e $\mathrm{C}_{4}$ ) $1 / 2$ Angus - $1 / 2$ Nelore versus $1 / 2$ Simental - 1/2 Nelore, na dieta A. Pela análise dos contrastes avaliados, observou-se que todos foram significativos $(\mathrm{P}<0,05)$. Assim, verificou-se que, independente da dieta, os animais mes-

Tabela 2 - Médias de quadrados mínimos para eficiência bionutricional, de acordo com grupo genético e dieta

Table 2 - Least square means for bio-nutritional efficiency

\begin{tabular}{|c|c|c|}
\hline \multirow[t]{2}{*}{$\begin{array}{l}\text { Grupo genético } \\
\text { Genetic group }\end{array}$} & \multicolumn{2}{|c|}{$\begin{array}{c}\text { Dieta* }^{*} \\
\text { Di]et }\end{array}$} \\
\hline & A & $\mathrm{B}$ \\
\hline Nelore & 299,70 & 429,74 \\
\hline Nellore & & \\
\hline $\begin{array}{l}1 / 2 \text { Angus - } 1 / 2 \text { Nelore } \\
1 / 2 \text { Angus - 1/2 Nellore }\end{array}$ & 371,81 & 529,84 \\
\hline $\begin{array}{l}1 / 2 \text { Simental - } 1 / 2 \text { Nelore } \\
1 / 2 \text { Simmental - 1/2 Nellore }\end{array}$ & 380,39 & 451,09 \\
\hline
\end{tabular}
according to genetic group and diet

A - Feno de Tanzânia mais $600 \mathrm{~g}$ de grão de soja moído.

B - Feno de Tanzânia + concentrado (65\% de grão de milho triturado, $11 \%$ de farelo de soja, $20 \%$ de farelo de algodão, $2,2 \%$ de calcário calcítico, 2,2\% de uréia e 40 gramas de rumensin).

A - Tanzânia Hay $+600 \mathrm{~g}$ of soybean meal.

B - Tanzânia Hay + concentrate ( $65 \%$ of ground corn, $11 \%$ of soybean meal, $20 \%$ of cottonseed meal, $2.2 \%$ lime, $2.2 \%$ of urea and $40 \mathrm{~g}$ of rumensin). tiços apresentaram melhor eficiência bionutricional do que os animais Nelore. Na dieta A, as médias das eficiências bionutricionais foram iguais a 376,10 e 299,70 para mestiços e Nelore, respectivamente. Nesta mesma seqüência, as médias, para a dieta B foram iguais a 490,46 e 429,74. É importante ressaltar que, quando se analisou a conversão alimentar, não houve efeitos significativos da interação $(\mathrm{P}>0,05)$ entre grupo genético e dieta. Tampouco houve diferenças $(P>0,05)$ entre os grupos genéticos. Nesse caso, as médias de conversão alimentar foram 14,15; 14,24 e $14,30 \mathrm{~kg}$ de MS consumida/kg de ganho de peso vivo para $\mathrm{N}$, AN e $\mathrm{SN}$, respectivamente. As conversões alimentares foram diferentes $(\mathrm{P}<0,05)$ para as dietas A e B, com médias iguais a 20,02 e 8,44, respectivamente.

Estes resultados, especialmente aqueles relativos aos desempenhos na dieta de baixa qualidade nutricional, estão aparentemente em discordância com vários outros encontrados na literatura (MOORE et al., 1975; MANZANO et al., 1986; VALADARES FILHO, 1987; OLIVEIRA et al., 1991). Esses autores observaram que, aparentemente, os zebuínos demonstraram melhor capacidade de utilização de dietas de baixa qualidade do que animais mestiços com raças européias. De fato, HUNTER e SIEBERT (1985), trabalhando com animais das raças Brahman e Hereford consumindo dietas de forragem contendo, aproximadamente, $3 \%$ de proteína bruta, observaram haver diferenças entre zebuínos e taurinos e sugeriram que a superioridade dos zebuínos se devia às maiores concentrações de uréia plasmática e de amônia ruminal. Provavelmente, nas condições do presente experimento, a correção do nível de proteína bruta para, aproximadamente, $6 \%$, com a adição do grão de soja moído, tenha eliminado a vantagem que os animais Nelore teriam na dieta A.

No entanto, os resultados do presente trabalho estão de acordo com observações e avaliações de desempenho de animais F1s em pastagens. Nessas condições, animais "meio-sangue" de raças européias com o Nelore têm apresentado superioridade de, aproximadamente, $15 \%$ em relação aos nelores (EUCLIDES FILHO, 1997).

Além disso, essa melhoria da dieta associada à eliminação de estresses causados por parasitas e doenças e pela insolação pode, de acordo com FRISCH e VERCOE (1977), fazer com que as diferenças entre os desempenhos de grupos genéticos diversos estejam altamente correlacionadas com o consumo voluntário. As médias observadas para os consumos 
diários médios, na dieta A, foram 6,77 e 7,94 kg de MS/dia para Nelore e F1s, respectivamente (Tabela 3).

A comparação entre os animais "meio-sangue", por outro lado, revelou que os desempenhos dos animais foram dependentes da dieta. Animais 1/2 Angus - $1 / 2$ Nelore apresentaram desempenho superior aos verificados para os $1 / 2$ Simental - $1 / 2$ Nelore na dieta B $(529,84$ versus 451,09$)$, enquanto na dieta A não houve diferença entre estes grupos genéticos ( 371,81 versus 380,39 , respectivamente). Analisando-se os ganhos diários médios de peso, no período, e os consumos diários médios de matéria seca verifica-se que, na dieta $\mathrm{B}$, os animais $\mathrm{F} 1 \mathrm{~s}$, $\mathrm{AN}$ e $\mathrm{SN}$, além de apresentarem o mesmo ganho $(0,87 \mathrm{~kg})$, apresentaram consumo de MS semelhante (7,94 kg de MS/dia, em média) (Tabela 3). Apesar de as diferenças nos requerimentos nutricionais, para mantença, existentes entre animais Angus e Simental, sugeridas pelo NATIONAL RESEARCH COUNCIL - NRC (1996), não terem sido evidenciadas nos animais F1s resultantes de cruzamentos dessas raças com a Nelore, quando se analisaram o ganho de peso e o consumo independentemente, as diferenças observadas na EBN podem estar sugerindo que elas realmente existem e a análise bivariada é, de fato, um método mais eficiente para sua discriminação. Isto que estaria de acordo com o reportado por GUIDONI (1994).

A eficiência na utilização de alimentos, além de poder variar com o grupo genético do animal, está de acordo com vários resultados de pesquisa, dependente do ponto de avaliação utilizado (tempo pré-definido, peso fixo e grau de terminação). Nesse caso, como se adotou a espessura de gordura subcutânea como ponto de avaliação dos animais, era de se esperar que os animais "meio-sangue" Angus-Nelore fossem mais eficientes. Isso porque estes animais, nestas condições, necessitariam de ser mantidos por um período menor, uma vez que deveriam atingir o ponto de terminação mais rapidamente. De fato, de acordo com os resultados de GREGORY et al. (1994), animais filhos de reprodutores Angus e Hereford foram mais eficientes que os resultantes de acasalamentos com touros de raças européias continentais, quando esses foram avaliados ao mesmo escore de marmoreio ou mesma espessura de gordura. Isto se deve ao fato que, apesar de os animais SN estarem recebendo a mesma ração que os demais, estes permaneceram mais tempo em alimentação para atingirem o ponto de acabamento pré-determinado. Para a dieta B, enquanto para os animais SN foram necessários, em média, 484 dias em alimentação, os nelores permaneceram 414 e os mestiços AN 407 dias. URICK et al. (1991) também observaram que, para a situação em que a avaliação dos animais ocorria a uma espessura de gordura pré-definida, animais filhos de pais Angus e mães Hereford permaneceram muito menos tempo em alimentação do que filhos de touros Simental, Pinzgauer e Tarantaise quando foram acasalados com fêmeas dessa mesma raça. Por outro lado, quando esses autores consideraram como ponto de avaliação o peso fixo de $400 \mathrm{~kg}$, ou mesmo, o tempo prédeterminado de 382 dias, verificou-se, para conversão alimentar, melhor desempenho para as progênies de touros Simental. Quando o ponto de avaliação foi definido pela espessura de gordura, os "meio-sangue" Angus apresentaram melhor conversão alimentar.

Outros resultados indicam que a eficiência na utilização de alimentos não é influenciada pelo grupo genético. EUCLIDES FILHO et al. (1997), avaliando o desempenho de animais Nelore e seus mestiços

Tabela 3 - Médias de quadrados mínimos para ganho de peso diário médio durante o período experimental (kg/dia), consumo diário médio matéria seca (kg/dia) e consumo diário de matéria seca/100 kg PV (C100), de acordo com o grupo genético e dieta

Table 3 - Least square means for average daily gain during the experimental period ( $\mathrm{kg}$ ), average daily dry matter intake ( $\mathrm{kg}$ ) and average daily dry matter intake/100 kg LW (C100), according to genetic group and diet

\begin{tabular}{|c|c|c|c|c|c|c|}
\hline \multirow[t]{2}{*}{$\begin{array}{l}\text { Grupo genético } \\
\text { Genetic group }\end{array}$} & \multicolumn{3}{|c|}{$\begin{array}{c}\text { Dieta A } \\
\text { Diet A }\end{array}$} & \multicolumn{3}{|c|}{$\begin{array}{l}\text { Dieta B } \\
\text { Diet B }\end{array}$} \\
\hline & $\begin{array}{l}\text { Ganho de peso } \\
\text { Weight gain }\end{array}$ & $\begin{array}{l}\text { Consumo } \\
\text { Intake }\end{array}$ & C100 & $\begin{array}{c}\text { Ganho de peso } \\
\text { Weight gain }\end{array}$ & $\begin{array}{l}\text { Consumo } \\
\text { Intake }\end{array}$ & $\mathrm{C} 100$ \\
\hline $\begin{array}{l}\text { Nelore } \\
\text { Nellore }\end{array}$ & 0,19 & 3,59 & 1,73 & 0,74 & 6,77 & 2,15 \\
\hline $\begin{array}{l}1 / 2 \text { Angus }-1 / 2 \text { Nelore } \\
1 / 2 \text { Angus }-1 / 2 \text { Nellore }\end{array}$ & 0,22 & 4,54 & 2,02 & 0,85 & 8,05 & 2,10 \\
\hline $\begin{array}{l}1 / 2 \text { Simental - } 1 / 2 \text { Nelore } \\
1 / 2 \text { Simmental - } 1 / 2 \text { Nellore }\end{array}$ & 0,24 & 4,65 & 1,92 & 0,89 & 7,83 & 2,11 \\
\hline
\end{tabular}


com Charolês, Chianina e Fleckvieh em diferentes dietas, não observaram diferenças entre os diversos grupos genéticos estudados. Houve, todavia, diferenças para conversão alimentar dos animais, em função da dieta, sendo superior na dieta de maior densidade energética. É importante ressaltar que, nesse caso, o ponto de avaliação adotado foi peso final pré-definido.

Divergências entre resultados envolvendo avaliações de eficiências relacionadas à ingestão e digestão de alimentos, além de serem influenciadas pelos chamados pontos de avaliação, são também dependentes do conjunto de características utilizadas para comparação. Dessa forma, os resultados podem variar, caso as características utilizadas sejam mensurações realizadas nos animais vivos ou na carcaça.

McKAY e RAHNEFELD (1988), estudando a eficiência de utilização de alimentos em animais mestiços envolvendo touros das raças continentais Charolês, Simental, Limousin e Chianina acasalados com vacas mestiças de várias raças, verificaram que os resultados foram dependentes da base de comparação. Enquanto não houve diferenças importantes entre os grupos genéticos, quando as avaliações foram baseadas em medidas realizadas em animais vivos (peso vivo ou ganho de peso), essas foram significativas, quando as comparações foram conduzidas com base em características de carcaça como, peso de carcaça quente, peso de músculo, peso de gordura ou peso de ossos.

\section{Conclusões}

Na dieta de melhor qualidade (dieta B), os animais $1 / 2$ Angus - $1 / 2$ Nelore apresentaram melhor eficiência bionutricional do que os $1 / 2$ Simental - 1/2 Nelore. Essa diferença não foi observada na dieta de baixa qualidade (dieta $\mathrm{A}$ ).

Animais mestiços, independente da dieta, apresentaram melhores desempenhos do que animais Nelore.

A análise multivariada, além de mostrar-se adequada às comparações de desempenho nutricional, foi capaz de discriminar os diferentes tratamentos.

\section{Referências Bibliográficas}

CEZAR, I.M., EUCLIDES FILHO, K. 1996. Novilho precoce: reflexos na eficiência e economicidade do sistema de produção. Campo Grande: EMBRAPA-CNPGC. 31p. (EMBRAPACNPGC. Documentos, 66).

EUCLIDES FILHO, K. 1997. A pecuária de corte no Brasil: novos horizontes, novos desafios. Campo Grande: EMBRAPA - CNPGC. 28p. (EMBRAPA - CNPGC. Documentos, 69).
EUCLIDES FILHO, K., EUCLIDES, V.P.B., FIGUEIREDO, G.R. et al. 1997. Avaliação de animais Nelore e seus mestiços com Charolês, Fleckvieh e Chianina, em três dietas. 1. Ganho de peso e conversão alimentar. R. Soc. Bras. Zootec., 26(1):66-72.

FRISCH, J.E., VERCOE, J.E. 1977. Food intake, eating rate, weight gains, metabolic rate and efficiency of feed utilization in Bos taurus and Bos indicus crossbred cattle. Anim. Prod., 24(1):343-358.

GONÇALVES, L.C., SILVA, J.F.C., ESTEVÃO, M.M. et al. 1991. Consumo e digestibilidade da matéria seca e da energia em zebuínos, taurinos, seus mestiços e bubalinos. R. Soc. Bras. Zootec., 20(4):384-395.

GREGORY, K.E., CUNDIFF, L.V., KOCH, R.M. 1994. Breed effects, dietary energy density effects, and retained heterosis on different measures of gain efficiency in beef cattle. J. Anim. Sci., 72(5):1138-1154.

GUIDONI, A.L. Alternativas para comparar tratamentos envolvendo o desempenho nutricional animal. Piracicaba, SP: ESALQ/USP, 1994. 105p. Tese (Doutorado) - Escola Superior de Agricultura "Luiz de Queiroz", 1994.

HUNTER, R.A., SIEBERT, B.D. 1985. Utilization of lowquality roughage by Bos taurus and Bos indicus cattle. 2. The effect of rumen-degradable nitrogen and sulphur on voluntary food intake and rumen characteristics. Brit. J. Nut., 53(3):649-656.

LEDGER, H.P., ROGERSON, A., FREEMAN, G.H. 1970. Further studies on the voluntary food intake of Bos indicus, Bos taurus and crossbred catle. Anim. Prod., 12(3):425-432.

MANZANO, A., NOVAES, N.J., ESTEVES, S.M. Eficiência de utilização de nutrientes pelas raças Nelore e Canchim e mestiços Nelore-zebu. In: REUNIÃO ANNUAL DA SOCIEDADE BRASILEIRA DE ZOOTECNIA, 23, 1986, Campo Grande. Anais... Campo Grande: SBZ, 1986. p.117.

McKAY, R.M., RAHNEFELD, W.G.W. 1988. Efficiency of feed utilization by three-breed cross steers. Can. J. Anim. Sci., 68(1):93-106.

MINSON, D.J. 1990. Forage in ruminant nutrition. 1.ed. San Diego: Academic Press. 483p.

MOORE, R.L., ESSIG, H.W., SMITHSON, L.F. 1975. Influence of breeds of beef cattle on ration utilization. J. Anim. Sci., 41(1):203-207.

NATIONAL RESEARCH COUNCIL - NRC. 1996. Nutrient requirements of beef cattle. 7.ed. Washington: National Academy Press. 242p.

OLIVEIRA, R.F.M., FONTE, C.A.A., SILVA, J.F.C. 1991. Consumo e digestibilidade de dietas com duas proporções de concentrados fornecidas a bovinos de três grupos genéticos. R. Soc. Bras. Zootec., 20(5):513-521.

SAS Institute (Cary, USA). SAS/STAT User's guide, version 6. 4. ed. Cary, 1989, v.1, 943p.

URICK, J.J., MAcNEIL, M.D., REYNOLDS, W.L. 1991. Biological type on postweaning growth, feed efficiency and carcass characteristics of steers. J. Anim. Sci., 69(2):490-497.

VALADARES FILHO, S.C., SILVA, J.F.C., LEÃO, M.I. et al. 1987. Estudo comparativo da digestão de matéria seca e carboidratos em bovinos e bubalinos alimentados com diferentes rações. R. Soc. Bras. Zootec., 16(2):120-130.

WRIGHT, A. 1971. Farming systems, models and simulation. In: DENT, J.B., ANDERSON, J.R. (Eds.) Systems analysis in agricultural management. London: John Wiley. p.17-33.

Recebido em: 08/02/00

Aceito em: 21/09/00 